\title{
Religiosidade e espiritualidade na prática clínica em saúde mental
}

\author{
Religiosity and spirituality in mental health clinical practice
}

Religiosidad y espiritualidad en la práctica clínica de salud mental

\begin{abstract}
Deisiane Duarte Rodrigues ${ }^{1 *}$, Rayziane Christiele de Freitas Fonseca ${ }^{1}$, José Ricardo Ferreira da Fonseca ${ }^{1}$, Rebeca Caranha Araújo ${ }^{1}$, Lara Abreu Ribeiro Alves ${ }^{1}$, Susy Cavalcante Harjani ${ }^{1}$, Henry Walber Dantas Vieira ${ }^{1}$.
\end{abstract}

\section{RESUMO}

Objetivo: Avaliar a influência da religiosidade e espiritualidade no campo da saúde mental por meio dos profissionais de saúde. Métodos: estudo com abordagem qualiquantitativo e descritivo-exploratória com 7 profissionais de saúde, em um Centro de Atenção Psicossocial no município de Manaus-AM. Foi realizado junto com os profissionais no CAPS um questionário e após essa etapa, a análise dos dados. Resultados: A religiosidade e espiritualidade pode gerar influências positivas em aspectos relevantes para a saúde. Portanto, depende da forma como cada paciente conduz, utiliza e interpreta essas práticas durante a sua reabilitação, por isso é importante a presença dos profissionais de saúde, para agregar esse elo de religiosidade, espiritualidade e saúde de forma saudável. Conclusão: O conteúdo religiosidade e espiritualidade pode ter um impacto positivo na saúde de pessoas com transtornos mentais e pode ajudá-las na adesão ao tratamento e nas dificuldades geradas pela doença. O número pequeno da amostra resultou pela indisponibilidade dos profissionais, sendo necessário ser produzido em mais unidades do CAPS.

Palavras-chave: Saúde mental, Espiritualidade, Religião

\section{ABSTRACT}

Objective: To evaluate the influence of religiosity and spirituality in the field of mental health through health professionals. Methods: a study with a qualitative and descriptive-exploratory approach with 7 health professionals, in a Psychosocial Care Center in the city of Manaus-AM, a questionnaire was applied and, after this stage, data analysis was carried out. Results: Religiosity and spirituality can generate positive influences in aspects relevant to health. Therefore, it depends on how each patient conducts, uses and interprets these practices during their rehabilitation, so it is important the presence of health professionals, to add this link religiosity, spirituality and health in a healthy way. Conclusion: The content religiosity and spirituality, can have a positive impact on the health of people with mental disorders and can help them in adhering to treatment and in the difficulties generated by the disease. The small sample size resulted from the unavailability of professionals, and it was necessary to be produced in more units of CAPS.

Key words:Mental health, Spirituality, Religion.

\section{RESUMEN}

Objetivo: evaluar la influencia de la religiosidad y la espiritualidad en el campo de la salud mental a través de profesionales de la salud. Métodos: estudio con enfoque cualitativo-descriptivo-exploratorio con 7 profesionales de la salud, en un Centro de Atención Psicosocial en la ciudad de Manaus-AM, se aplicó un

\footnotetext{
${ }^{1}$ Universidade Federal do Amazonas (UFAM), Manaus - Amazonas.

*E-mail: Deisianeduart@gmail.com
} 
cuestionario y, luego de esta etapa, se realizó el análisis de datos. Resultados:la religiosidad y la espiritualidad pueden generar influencias positivas en aspectos relevantes para la salud. Por lo tanto, depende de cómo cada paciente realice, use e interprete estas prácticas durante su rehabilitación, por lo que es importante la presencia de profesionales de la salud, para agregar este vínculo de religiosidad, espiritualidad y salud de una manera saludable. Conclusión: El contenido de religiosidad y espiritualidad puede tener un impacto positivo en la salud de las personas con trastornos mentales y puede ayudarles a adherirse al tratamiento y a las dificultades generadas por la enfermedad. El pequeño número de la muestra resultó de la falta de disponibilidad de profesionales, y fue necesario producirlo en más unidades de CAPS.

Palabras clave: Salud mental, Espiritualidad, Religión.

\section{INTRODUÇÃO}

Algumas pesquisas demonstram que a associação entre religiosidade, espiritualidade e saúde mental, proporciona ao indivíduo maior bem-estar, equilíbrio, adesão ao tratamento e enfrentamento da doença, através das práticas religiosas e espirituais. (YUNTA RE, 2016; SALIMENA AMO, 2016; ZERBETTOSR, 2017). Nota-se a importância dessa temática no meio científico e dos resultados que ela pode proporcionar na saúde, atuando como terapêutica complementar na prática clínica em saúde mental. Por isso é necessário entender os conceitos dos termos religiosidade e espiritualidade, para compreender que elas podem ter influências positivas ou negativas no que diz respeito ao tratamento do indivíduo com transtornos mentais. O termo religiosidade envolve uma dimensão complexa de condutas, práticas e relações socioculturais, envolvendo a união do indivíduo com a fé (ZERBETTO SR, et al., 2017). A espiritualidade é caracterizada por sentimentos intrínsecos, que abrange questões da existência humana e do seu relacionamento direto e indireto com o universo (LONGUINIERE ACF, et al., 2018).

Neste sentido, as práticas religiosas tornam-se relevante e encontram certo significado no momento em que $\mathrm{o}$ indivíduo se vê portador de alguma doença e em situações ameaçadoras de vida, fato que faz o individuo recorrer ao ambiente religioso como método de esperança, conforto e cura (ROCHA TP, et al., 2016). Manifesta-se também a espiritualidade, o indivíduo com problemas mentais começa a questionar-se sobre a razão de viver, surgindo uma ligação maior ou mais distante com Deus (YUNTA RE, 2016).

A influência da religiosidade e espiritualidade aos transtornos psiquiátricos pode desempenhar papel favorável ou não à saúde, essas práticas podem promover valores morais, melhorar a autoestima e proporcionar um sentimento de cura. Por outro lado, podem estar ligados com razões intrínsecas de culpa, diminuição do bem-estar, neurose e ideação psicótica. Em alguns contextos culturais é comum as pessoas acharem que a doença mental é castigo ou tem relação com espíritos malignos (YUNTA RE, 2016). Por isso, o profissional de saúde mental deve respeitar, reconhecer as escolhas e os aspectos socioculturais dos usuários, que muitas vezes são desconhecidos pelos profissionais, por estes centrarem os cuidados no modelo biomédico, focado na doença (SALIMENA AMO, et al., 2016).

A relação dos profissionais de saúde com a temática pode auxiliar a desenvolver vínculos com os usuários, para isso é necessário aprofundar o conhecimento ainda na formação acadêmica, oferecer no ambiente de trabalho capacitação voltada para a temática e ampliar mais pesquisas. A presença do conteúdo religiosidade e espiritualidade requer que os profissionais de saúde tenham conhecimento, saibam identificar e reconhecer o papel que exerce no campo da saúde mental no tratamento e na qualidade de vida dos usuários. Nesse sentido, estabeleceu-se para esse estudo o objetivo de avaliar a influência da religiosidade e espiritualidade no campo da saúde mental por meio dos profissionais de saúde.

\section{MÉTODOS}

Trata-se de uma pesquisa com abordagem qualiquantitativo e descritiva-exploratória, realizado em um Centro de Atenção Psicossocial (CAPs III), do município de Manaus-AM, vinculado à Secretaria Municipal de Saúde (SEMSA). 
Os critérios de inclusão foram: Profissionais de saúde que atuam no (CAPs III). Os critérios de exclusão foram: Profissionais afastados do serviço por qualquer natureza no período de coleta de dados, estagiários e residentes. A população estimada da pesquisa foi de 12 participantes, deste total apenas 7 compuseram a amostra, 2 não aceitaram participar da pesquisa, o motivo foi a falta de tempo para a entrevista, 3 profissionais não foram abordados por indisponibilidade de tempo dos mesmos em receber os pesquisadores, pois estavam em atendimento aos pacientes.

Os dados foram coletados no período de março a maio de 2019 , por meio da aplicação de um questionário composto por duas partes: Parte 1 - Perfil do participante; e parte 2 - Informações relacionadas à religiosidade e espiritualidade do participante da pesquisa. Os profissionais foram selecionados por amostragem aleatória por conveniência. Para análise de dados, utilizou-se o método de análise de conteúdo sugerido por Bardin (1977), por se tratar de uma técnica de análise de dados por meio de categorizações, o qual nos permite compreender as atitudes, opiniões, valores e crenças dos indivíduos a respeito das diversas questões presenciadas no cotidiano através dos dados coletados, obedecendo às etapas que seguem: 1) A pré-análise; 2) A exploração do material e, por fim 3) $O$ tratamento dos resultados.

Todos os participantes da pesquisa tiveram acesso primeiramente ao preenchimento do Termo de Consentimento Livre e Esclarecido (TCLE), honrando todas as providências em relação à dimensão ética da Resolução 466/2012 do Conselho Nacional de Saúde - CNS. A pesquisa foi autorizada sob o número do CAAE: 04702218.0.0000.5020, respeitando as normas éticas de anonimato, não maleficência, beneficência e justiça.

\section{RESULTADOS/DISCUSSÃO}

Os profissionais que aceitaram participar da pesquisa foram caracterizados, conforme a tabela abaixo, (Tabela 1). Participaram deste estudo 7 profissionais de saúde de diversas áreas, com idade entre 30 e 52 anos, sendo a maioria dos profissionais do sexo feminino. Todos relataram possuir afiliação religiosa, com destaque para a religião Católica que teve maior predomínio, seguida da religião Evangélica. $O$ tempo de trabalho no CAPS variou de 2 a 7 anos. A partir das entrevistas, observou-se que a religiosidade e espiritualidade se faz presente na vida e no cotidiano dos profissionais, uns com rotina semanal de ir ao templo religioso, outros mensalmente.

Tabela 1 - Caracterização dos profissionais de saúde do CAPS III.

\begin{tabular}{|c|c|c|c|}
\hline Idade & Sexo & Religião & Profissão \\
\hline 32 & $\mathrm{~F}$ & Evangélica & Nutricionista \\
\hline 30 & $\mathrm{~F}$ & Católica & Psicóloga \\
\hline 52 & $\mathrm{~F}$ & Evangélica & Psicóloga \\
\hline 33 & $\mathrm{M}$ & Católico & Farmacêutico \\
\hline 38 & $\mathrm{~F}$ & Católica & Educadora Física \\
\hline 43 & $\mathrm{~F}$ & Evangélica & Enfermeira \\
\hline 43 & $\mathrm{M}$ & Católico & Servidor publico \\
\hline
\end{tabular}

Fonte: Rodrigues DD, et al., 2020.

\section{Religiosidade/espiritualidade como suporte no regime terapêutico}

A maioria das pessoas atribuem um valor significativo as práticas religiosas e espirituais devido as questões culturais, a representatividade e os seus significados, manifestando-se de diferentes formas e possuindo fatores em comum como as suas contribuições na vida e na saúde das pessoas, sobretudo, na saúde mental, sendo raro encontrar casos em que essas práticas não se faz presente, tampouco tenha valor (ALVES GD, ASSIS RM, 2015). Dessa forma, a maior parte dos profissionais declarou acreditar em 
Deus e que a escolha da afiliação religiosa, sucedeu por influência dos pais, dos valores que essas práticas significam para eles.

"Me considero religiosa, sigo os mandamentos, fui criada numa família católica praticantes de ir à igreja, participei de catequese. Acredito em Deus desde sempre". (E1)

"Sou católico, frequento a igreja, participo de alguns grupos e vou semanalmente ao ambiente religioso, sempre acreditei na presença de Deus". (E4)

No presente estudo, quando os profissionais foram questionados se a religiosidade e a espiritualidade auxiliavam o tratamento de modo positivo ou negativo, a maioria respondeu que depende do momento em que o paciente se encontra e da forma como ele encara essas práticas.

O paciente pode se encontrar no momento de delírio, alucinações e psicose, que pode ser prejudicial devido ao comportamento e o pensamento desorganizado, em certos casos negando a necessidade do esquema terapêutico. Porém, por outro lado pode estar internamente preparado e motivado a aderir ao tratamento e comportamentos saudáveis para obter resultados positivos na saúde (ZERBETTO SR, et al., 2017).

\begin{abstract}
"Essa questão é uma faca de dois gumes, porque as vezes ela trabalha do lado positivo, trazendo maiores benefícios e esperança, mas tem o lado negativo de ele dizer que Deus vai curar, que não precisa da medicação e somente indo à igreja e orando vai ser abençoado com saúde, então tem essas duas vertentes". (E6)

"Depende do paciente, acho que a religiosidade/espiritualidade ajuda de forma negativa quando eles acham que não tomando o medicamento eles vão ser curados, e pode ser de forma positiva quando empregam a religiosidade/espiritualidade como tratamento auxiliar, mesmo tomando a medicação". (E7)
\end{abstract}

"Aqueles pacientes que se apegam com Deus no seu momento de sofrimento, conseguem ter uma melhora mais significativa, eu vejo como algo positivo. Diferente daqueles que não acreditam na existência de um ser maior, eles acabam tendo uma vida de forma mais amarga, tudo mais dificultoso e quando pensam em Deus é como se fosse um ser que castiga, que quer vê-lo sofrer". (E1)

Compreende-se a complexidade destas narrativas e a importância delas para o fortalecimento de estratégias voltadas ao benefício da saúde e as necessidades do paciente. Desta forma, a fé e a oração, produz fortalecimento nos momentos angustiantes e turbulentos e muitos pacientes conseguem lidar melhor nos momentos difíceis, gerando resultados positivos.

Um estudo realizado em um Centro de Atenção Psicossocial-Álcool e Drogas (CAPS AD) com usuários diagnosticado com dependência de álcool, demonstrou que a religiosidade e espiritualidade influência de modo positivo a vida e o tratamento dos dependentes de álcool, através do conforto de palavras, orações e ritos, promovendo mudanças na rotina, nos hábitos e no comportamento dos usuários (ZERBETTO SR, et al., 2017).

Os profissionais evidenciaram nos depoimentos que já houve experiências com pacientes que demonstravam abordagens de cunho religiosos de forma fanática e as crises tornaram-se mais frequentes com discurso que ("Deus vai curar") resultando no abandono do tratamento e esperança do paciente por solução divina.

É comum casos em que o sujeito e a família recorrem primeiro em ambientes religiosos como forma de tratamento, algumas atividades religiosas acabam expondo o sujeito como alguém possuído por entidade do mal, o que contribui para isolamento social e agravamento do quadro sintomático (REINALDO MAS, SANTOS RLF, 2016). 
Outra abordagem realizada entre os profissionais, trata-se da religiosidade e espiritualidades, se ajuda de certa forma o paciente e a família a enfrentar melhor a doença, alguns profissionais responderam com o mesmo dialogo, na maioria dos casos nota-se uma capacidade maior de lidar com a doença e quando a família se põe no lugar do doente e observa que a sua presença no ambiente religioso traz resultados positivos, ela encara com outros olhares e com maior esperança.

"Ajuda o paciente e a família a enfrentar melhor a doença e teve muitos casos que igreja assumiu papel de cuidado, então hoje já não se vê isso com tanta estranheza". (E2)

"Depende muito da visão que a família tem das práticas religiosas e espirituais, se for de forma saudável acredito que sim. Mas na maioria dos casos o que se percebe é sua ajuda de forma positiva". (E3)

É importante evidenciar que o ambiente religioso pode fazer sua parte como forma de ajudar no enfretamento das dificuldades impostas pela doença, atuando como terapêutica complementar (ZERBETTO SR, et al., 2017).

Porém, a família e o paciente não podem deixar como último plano as questões positivas que a psicoterapia, o regime terapêutico e o acompanhamento de uma equipe multiprofissional podem trazer para o benefício da saúde. Salienta-se que o ambiente religioso não pode assumir sozinho o papel de cuidado.

Diante das declarações dos profissionais, a religiosidade e a espiritualidade podem gerar influências positivas em aspectos relevantes para a saúde, pode atuar como apoio complementar para a família e o paciente diante das dificuldades impostas pela doença.

No entanto, depende da forma como cada paciente conduz e utiliza essas práticas durante a sua reabilitação, por isso é importante a ajuda dos profissionais para agregar esse elo religiosidade, espiritualidade e saúde de forma saudável e oferecer suporte para que previna situações negativas que possa interferir e conduzir consequências na saúde e na vida do paciente.

\section{Conhecimento dos profissionais na abordagem religiosa e espiritual}

Durante a entrevista os profissionais de saúde foram questionados se possuem dificuldades em abordar questões religiosas e espirituais durante a prática assistencial. A maioria relatou não haver barreira e que sempre são abordados para tratarem desses assuntos.

Alguns profissionais podem ter dificuldades em abordar estas questões junto ao paciente e não se sentir confortável, assim como acreditar que a religiosidade e espiritualidade não tem influência no processo de adoecimento e de tratamento (REINALDO MAS, SANTOS RLF, 2016).

"Com o tempo, a gente vai aprendendo a lidar melhor com isso, por conta do viés, quando a gente percebe que uma pessoa traz muito forte essas questões, a gente não nega isso, faz parte da pessoa e hoje o próprioministério da saúde entende que há essa parte da religião e espiritualidade, não é mais só o biológico e se torna mais fácil de abordar nas nossas práticas". (E2)

"Eu não sinto dificuldades em abordar estas questões, mas eu sempre oriento no sentido de não parar a medicação e usar o conteúdo religiosidade e espiritualidade como alternativa". (E4)

"Não tenho dificuldade, até porque eu lido com isso de forma generalizada, eu não direciono para uma religião especifica, eu respeito a religiosidade que 0 paciente me traz, independente do que seja". (E3)

Os profissionais de saúde possuem experiência com pessoas de diferentes comportamentos, cabendo a ele dialogar de forma adequada e construir um relacionamento saudável com o paciente. O relacionamento interpessoal entre profissional e paciente deve ser fator primordial para promover empatia, confiança e uma 
boa comunicação, para que o pacientese sinta confortável ao dialogar com as questões da sua religiosidade e espiritualidade (SALIMENA AMO, et al., 2016).

Devemos salientar que para um bom diálogo é preciso compreender que o profissional de saúde precisa se entender e sobretudo, aprender sobre a sua religiosidade e espiritualidade (BORGES MS, SANTOS MBC, PINHEIRO TG, 2015). Para que dessa forma, ele possa sentir-se confortável ao abordar estas questões de forma satisfatória. $O$ treinamento de profissionais durante a assistência, com ênfase num cuidado espiritual oferecido aos pacientes, gera efeitos positivos e possibilita maior comunicação e confiança entre profissional e paciente (PAAL P, ROSER T, FRICK E, 2015).

Sabe-se que umas das principais barreiras para o relacionamento confiável é a falta de conhecimento, treinamento e desconforto dos profissionais com o tema (BORGES MS, SANTOS MBC, PINHEIRO TG, 2015). Acentua-se que os profissionais de saúde devem sobretudo respeitar os valores da religiosidade e espiritualidade do paciente e, precisam estar preparados para enfrentar as emoções e trabalhar no cuidado de forma integral, nas suas dimensões biológica, mental e espiritual do ser humano. Questionou-se aos profissionais se durante a prática assistencial o mesmo utiliza ou já utilizou meios relacionados à religiosidade e espiritualidade para prestar atendimento.

"Diretamente não, mas em algumas psicoterapias usamos mensagens de motivação, autoestima, de ânimo e gratidão, mostrando o mundo com outros olhos e as coisas boas da vida, isso acaba entrando no campo da espiritualidade. Quase sempre quando a gente leva essas mensagens eles trazem alguma coisa da bíblia ou da experiência deles religiosas e a gente abre espaço para que eles falem". (E2)

"Em algumas atividades do CAPS é utilizado mensagens religiosas e espirituais, os próprios usuários gostam e pedem para a gente cantar, o que se observa é que eles sempre agradecem a Deus e rezam". (E5)

O CAPS não possui psicoterapias ligadas a religiosidade e espiritualidade, mas abrem espaço para queestas questões sejam dialogadas, atuando como ajuda terapêutica. Desta forma, os profissionais precisam estar atentos para orientar e integrar o cuidado de forma com que o paciente não perca a esperança e nem abandone o tratamento.

A influência da espiritualidade e religiosidade na saúde física atua de maneira positiva, resultando na maioria dos casos em melhora no quadro de saúde, no enfretamento das situações difíceis e pensamentos negativos (INOUE TM, VECINA MA, 2017). Por isso a importância de enfatizar esta temática no cenário da saúde, seja em qualquer área, é evidente os benefícios. Em relação as interferências do conteúdo religioso no regime terapêutico, os profissionais afirmaram já ter acontecido alguns casos com os pacientes em abandono total do tratamento.

"Já teve casos em que o pastor disse ao paciente que não precisava tomar medicação, que a igreja ia curar, nesse caso a família veio pedir ajuda do CAPS. Houve inclusive situações em que o médico chamou o pastor para conversar com ele". (E6)

"Já teve relatos de pacientes que o pastor orou e disse que estava curado, tem pacientes que já foi internado em comunidade religiosa por práticas pra retiradas de demônios, a gente teve que chamar o pastor e a família para esclarecer e tirar dúvidas por causa dessa intervenção". (E2)

Os profissionais reforçam e orientam que Deus pode melhorar a situação de saúde do usuário, mas essa ajuda pode vim também através da medicação, psicoterapia e da ajuda dos profissionais.A família é um fator primordial para o processo de relação profissional-paciente o que possibilita a correta tomada de decisões, protegendo-o de situações quem coloquem sua vida em risco (REINALDO MAS, SANTOS RLF, 2016). No entanto é através dela que se tem esse acesso de informação quanto o estado físico e mental do paciente e o tipo de orientações que estão recebendo. 
O resultado demonstrou percepções positivas em relação a temática, os profissionais destacaram a influência desse conteúdo no processo de qualidade de vida dos pacientes e sua relevância nas intervenções em saúde, todos foram unanimes quanto a importância dessa temática para a pratica clínica em saúde mental.

\section{A importância de inserir o conteúdo religiosidade e espiritualidade na formação acadêmica}

Durante o diálogo com os profissionais, foi questionado se as relações entre saúde, religião e espiritualidade deve ser incluído nos currículos da área de saúde. Sabe-se que esta temática é pouco abordada durante a formação acadêmica, apesar dos estudos que se dedicam a pesquisar sobre essa relação (BORGES MS, 2015; REINALDO MAS, 2016; LONGUINIERE ACF, 2018).

"Poderia ser incluído durante a formação, principalmente para quem não tem tanta facilidade. Entrar nesse campo não quer dizer que está fugindo do biológico e da ciência. Pensando de outra forma essa realidade se torna mais difícil para quem é ateu, quando se tem um conhecimento que vem de berço é mais fácil de tentar remanejar, mas quando a pessoa é atéia se torna mais difícil". (E2)

"Acredito que sim, o tratamento tem que vim de todos os lados, seria interessante atuar em conjunto". (E5)

"Sim, na maioria dos casos esse conhecimento já vem de berço seria interessante abordar esta temática ainda na formação acadêmica, para o estudante saber lidar na pratica". (E1)

As informações escassas e imprecisas durante a formação acadêmica resultam como um fator negativo quando o profissional durante a pratica assistencial é abordado pelo paciente e pela família para tratar sobre esta temática, o cenário torna-se pior quando o profissional não possui afiliação religiosa e não considera esses aspectos durante a assistência (ABUCHAIM CSA, 2018; HENNING CM, 2015).

Sendo assim, torna-se importante criar espaços para o debate e abordagem dessa temática, seja em qualquer área da saúde e incentivar pesquisas ainda durante a vivência acadêmica.

Adquirir conhecimento e métodos de assistência que tratem da parte biopsicossocial e espiritual, não induz os acadêmicos e profissionais a abandonarem a ciência e tudo que foi aprendido durante $o$ ambiente universitário, mas incentiva a atuar de forma mais humana, compreendendo o paciente e dando todo o cuidado necessário.

Os serviços de saúde devem fornecer apoio complementar de recursos espirituais e religiosos para que se tenha um cuidado mais integral (SAAD M, et al., 2015). Essa associação nos serviços de saúde, tem o intuito de olhar para aqueles pacientes que as crenças religiosas e espirituais têm um valor mais significativo e no momento da hospitalização a sua associação será um indicador positivo no quadro de saúde.

No entanto, nota-se a importância de inserir na vivencia acadêmica as relações que a religiosidade e a espiritualidade contribui para a saúde, para que o profissional ao adentrar no mercado de trabalho já tenha um conhecimento adquirido durante a universidade, sabe-se o quão complexo esse assunto se torna na pratica, porém, a sua dimensão positiva no momento do sofrimento e no conforto da família se torna algo relevante para medicina, o cuidado e a pesquisa.

\section{CONCLUSÃO}

O estudo permitiu avaliar a percepção dos profissionais de saúde sobre a importância de inserir a religiosidade e espiritualidade como terapêutica complementar na prática clínica em saúde mental. Identificou-se que essa relação pode contribuir de forma positiva na vida de pessoas com transtornos mentais e pode ajudá-las a encarar com perspectiva de adesão e nas dificuldades geradas pela doença. Investigou-se que os profissionais de saúde não possuem dificuldade para abordar essas questões no 
ambiente de trabalho, nota-se que eles orientam a forma saudável do seu uso para a melhora do quadro de saúde, considerando em sua assistência a dimensão que a religiosidade e espiritualidade pode auxiliar em sua pratica assistencial, contribuindo no relacionamento interpessoal e reabilitação do paciente.

\section{REFERÊNCIAS}

1. ABUCHAIM CSA. Espiritualidade/religiosidade como recurso terapêutico na pratica clínica: concepções dos estudantes de graduação em medicina da Escola Paulista de Medicina. Dissertação (Mestrado e Ciências da Saúde) - Universidade Federal de São Paulo, Programa de Pós-graduação em Ensino em Ciências da Saúde, São Paulo, 2018.

2. ALVES GD, ASSIS RM. O desenvolvimento religioso e espiritual e a saúde mental: discutindo alguns de seus significados. Revista Conexões Psi, 2015; 3 (1): 72-100.

3. AMARANTE P, NUNES MO. A reforma psiquiátrica no SUS e a luta por uma sociedade sem manicômios. Ciênc. Saúde coletiva, 2018; 23(6): 2067-74.

4. BARDIN L. Análise de conteúdo. Tradução de Luis Antero Reto e Augusto Pinheiro. Lisboa: Edições 70, 1977.

5. BORGES MS, SANTOS MBC, PINHEIRO TG. Representações sociais sobre religião e espiritualidade. Rev Bras Enferm, 2015; 68(4): 609-16.

6. HENNING-GERONASSO CM, MORÉ OLC. Influência da Religiosidade/Espiritualidade no Contexto Psicoterapêutico. Psicologia: ciência e profissão, 2015, 35(3): 711-725.

7. INOUE TM, VECINA MA. Espiritualidade e/ou religiosidade e saúde: uma revisão de literatura. JHealth Sci Inst, 2017; 35 (2): 127-30.

8. LONGUINIERE ACF, YARID SD, SILVA ECS. Influência da religiosidade/espiritualidade do profissional de saúde no cuidado ao paciente crítico. Rev Cuid, 2018; 9(1):1961-72.

9. MELO CF, et al. Correlação entre religiosidade, espiritualidade e qualidade de vida: uma revisão de literatura. Estud. Pesqui. Psicol, 2015; 15(2): 447-464.

10. MOREIRA-ALMEIDA A, LUCCHETTI G. Panorama das pesquisas em ciência, Saúde e Espiritualidade. Ciência e Cultura, 2016; 68(1): 54-57.

11. NETO GV, et al. Spirituality review on mental health and psychiatric nursing. Rev Bras Enferm, 2018; 71(5): 232333.

12. PAAL P, ROSER T, FRICK E. Spiritual care training provided to healthcare professionals: a systematic review. J Pastoral Care Counsel, 2015; 69(1):19-30.

13. ROCHA TP, et al. A Influência da Espiritualidade e da Religiosidade no Tratamento Oncológico: percepção da pessoa com câncer. Rev. Tendên. da Enferm. Profis., 2016; 8(4): 2031-2036.

14. REINALDO MAS, SANTOS RLF. Religião e transtornos mentais na perspectiva de profissionais de saúde, pacientes psiquiátricos e seus familiares. Saúde Debate, 2016; 40(110):162- 171.

15. SAAD M, LUCCHETTI G, PERES MFP, MEDEIROSR. Toward the Concept of 'Spiritist Chaplaincy'. J Relig Health, $2015 ; 54: 1460-1469$.

16. SALIMENA AMO, et al. Compreensão da espiritualidade para os portadores de transtorno mental: contribuições para o cuidado de enfermagem. Rev Gaúcha Enferm, 2016; 37(3).

17. SILVA AH, FOSSÁ MIT. Análise de conteúdo: exemplos de aplicação da técnica para análise de dados qualitativos. Qualitas Revista Eletrônica, 2015; 17(1).

18. VANCINI LR, et al. The Spiritism as therapy in the health care in the epilepsy. Rev Bras, 2016; 69(4): 804-10.

19. YUNTA RE. Determinantes sociales de la salud mental. Rol de la religiosidad. Pers Bioét, 2016; 20(2): $192-204$.

20. ZERBETTO SR, et al. Religiosidade e espiritualidade: mecanismo de influência positiva sobre a vida e tratamento do alcoolista. Esc. Anna Nery, 2017; 21(1). 